\title{
Análisis físico-químico \\ de la relación señal-ruido en imágenes de resonancia magnética nuclear, IRMN
}

\section{Physical-chemical analysis of signal-noise ratio in nuclear magnetic resonance images, IRMN}

\section{Resumen}

En el ámbito clínico, una de las mayores preocupaciones se enfoca en cómo mejorar la calidad de las imágenes diagnósticas, para así, poder detectar patologías con mayor precisión; en el presente trabajo, los investigadores del área de imágenes diagnosticas del Grupo de Investigación y Desarrollo Tecnológico Aplicado (INDETECA), se enfocará en las imágenes de resonancia magnética, abordando el problema de la relación señal - ruido desde un punto de vista físico-químico, relacionando la señal y el ruido con las propiedades magnéticas de algunos núcleos, analizando también cómo el entorno físico químico afecta la señal. Para mejorar las imágenes se utilizó un filtrado en el espacio K y su posterior reconstrucción se realiza utilizando como método matemático la Transformada de Fourier.

\section{Palabras clave}

Imágenes de resonancia magnética nuclear, Transformada de Fourier, Relación Señal Ruido RSR.

Magíster en Ingeniería Química, Universidad Nacional de Colombia, Bogotá. Docente Investigador, Escuela Colombiana de Carreras Industriales, ECCl, área de Mecánica. Experto en el área térmica, energía sostenible y biocombustibles. Líder de la línea de Energía del Grupo de investigación en Aprovechamiento Tecnológico de Materiales y Energía, GIATME. E_mail: mmayorga@ecci.edu.co

** Físico, Universidad Industrial de Santander y candidato a Magíster en Física Médica, Universidad Nacional de Colombia, Sede Bogotá. Docente de Ingeniería Biodimédica, ECCl. Trabaja con el grupo de óptica y tratamiento de señales (GOST-UIS) en la realización de un correlador Vanderlugt. E_mail: wlvegav@ecci.edu.co

*** Físico, Universidad Distrital Francisco José de Caldas y candidato a Magíster en Física Médica de la Universidad Nacional de Colombia, Sede Bogotá. Docente investigador de la ECCl, área de Biomédica. Experto en protección radiológica para medicina nuclear. Participa en el grupo de investigación en Innovación y Desarrollo Tecnológico Aplicado, INDETECA. E_mail: esalazarc@ecci.edu.co 


\section{Abstract}

In the clinical setting of major concern focuses on how to improve the quality of diagnostic images, thus, to more accurately detect diseases within the diagnostic imaging group we will focus on the magnetic resonance images from this issue analysis arises from a physical standpoint on the variation of the chemical signal to noise ratio with the magnetic properties of some nuclei, in addition to analyzing how the environment affects the signal. Image enhancement filter used in the space $\mathrm{K}$ and subsequent reconstruction is performed using as the mathematical method the Fourier Transform.

\section{Keywords}

Magnetic Resonance Images, Fourier Transform, Relation Signal Noise (S/N).

\section{Introducción}

La técnica de diagnóstico por medio de Imágenes de Resonancia Magnética Nuclear, IRMN, se basa en el desdoblamiento de los estados energéticos de los núcleos cuando se aplica un campo magnético externo, dicho fenómeno se conoce comúnmente como efecto Zeeman [1]. La cantidad de desdoblamientos en los estados es dependiente del spin nuclear y esta propiedad solo se da en los núcleos con un número impar de protones ( $Z$ impar) o los núcleos con un número impar de neutrones ( $\mathrm{N}$ impar).

La Resonancia Magnética Nuclear (RMN) hace referencia a la excitación producida en el momento de dipolo magnético en el núcleo; mientras que si también se excitan los spines de los electrones se hablaría de resonancia magnética electrónica [2].

Dentro de todos los núcleos que cumplen el parámetro de $\mathrm{Z}$ o $\mathrm{N}$ impar, se selecciona el relacionado con el átomo de hidrógeno debido a su gran abundancia en el cuerpo humano y en tejidos biológicos, ya que está presente en las moléculas de agua, proteínas, etc. Es así como el núcleo del hidrógeno constituye la base de la señal presente en los radicales de los enlaces $O-H$ para el caso del agua y de los hidrógenos presentes en los radicales - $\mathrm{CH}_{2}-\mathrm{CH}_{3}$ para el caso de las grasas $[3,4]$.

Una vez se produce la magnetización en la materia, empieza un periodo de relajación de los núcleos hacia los estados energéticos más bajos, y es aquí donde los núcleos emiten RF que se capta a través de antenas. Estas RF dan cuenta del núcleo que las emite mediante la aplicación de técnicas denominadas secuencias, en las que se aplican unos gradientes de campo magnético durante los tiempos de relajación para desfasar las señales provenientes de los diferentes núcleos y así obtener la mejor información posible.

Ya lograda la codificación de la señal mediante pulsos de RF y gradientes de campo magnético, se requiere una etapa de decodificación de la señal mediante una transformación matemática que permita la posterior reconstrucción de la imagen; es aquí donde cobra importancia el presente trabajo, ya que a través de una buena relación señal-ruido, 
mediante un procesamiento de la señal, permite reconstruir una imagen mejorada sin alterar la información, dando así cuenta de alguna patología presente.

\section{Metodología}

\section{A. Proceso para la adquisición de la imagen}

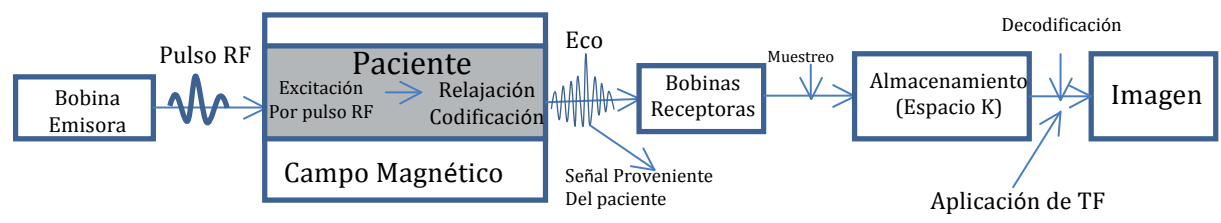

Figura. 1. Reelaborado a partir de [7]

La figura 1 ilustra todo el proceso para la adquisición de la imagen; se evidencia la aplicación de secuencias, codificación, espacio $\mathrm{K}$ y decodificación; en el caso de las secuencias, estas se aplican en la etapa denominada Paciente, en donde se efectúa una excitación los espines por RF y la codificación a partir de unos gradientes de campo magnético, lo que permite tener ecos más diferenciados.

Para la reducción del Ruido se usó un filtro de altura 20, con borde, para no dañar picos de la señal, la cual presentaba una intensidad máxima de 140; estos filtros aprovechan la condición de que, a altas frecuencias, el ruido presenta una mayor potencia que la misma señal.

\section{B. Construcción de Imágenes a través del espacio $K$}

Las señales provenientes de materia magnetizada se denominan ecos, los cuales se captan y se organizan mediante los gradientes de codificación de fase y de codificación de frecuencia. Una vez recogido el eco se codifica y se lleva a un espacio de manera ordenada para tener control de la información recibida, como se ilustra en la Figura 2. Después de este proceso, «... se transforma la señal que proviene de una situación espacial (dominio espacial) en una señal identificada por sus frecuencias espaciales (dominio frecuencial) mediante un proceso matemático denominado transformación de Fourier. En una secuencia clásica se recogen tantos ecos como codificaciones de fase; se observa que cada eco se identifica por la frecuencia espacial $K_{y}$ que le impone el gradiente de codificación de fase $G_{y}$. Estos ecos almacenados ordenadamente en forma digital y en formato de frecuencias espaciales constituyen lo que se denomina Espacio- $K$ » [7].

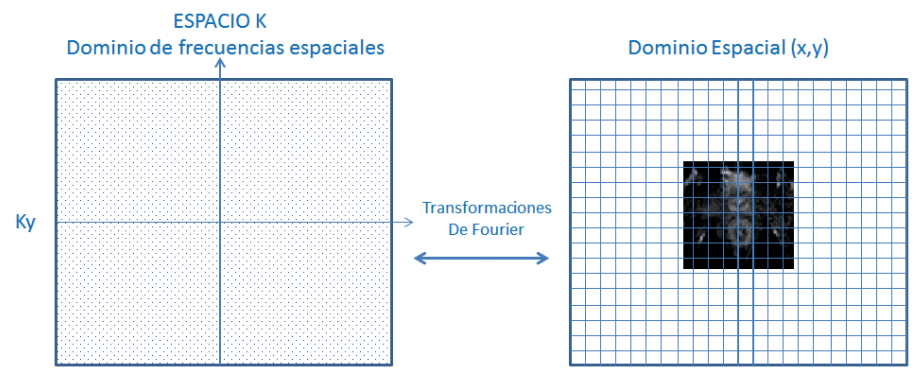

Figura 2. Ilustración del Espacio $\mathrm{K}$. 
La información del espacio $K$ es muy importante puesto que después de tener el dominio frecuencial, se puede aplicar una transformación inversa para ubicar espacialmente la frecuencia, asignarle un valor en una escala de gris o el color según la intensidad de la señal y permitir la formación de una imagen.

Uno de los procesos para depurar las frecuencias que se generan por la interacción entre las radiofrecuencias, RF, y que pueden afectar la calidad de las imágenes se presenta en los siguientes apartados.

\section{La Transformada de Fourier}

En la mayoría de equipos de imágenes que usan $\mathrm{RMN}$, se usan satisfactoriamente filtros basados en la Transformada de Fourier, tales como los filtros Hanning, Hamming, Kalman, etc. Dado que las imágenes se obtienen a partir del espacio $K$ y en el dominio de la frecuencia, el método utilizado para reconstruir la imagen es realizar una transformada de Fourier, en la que se debe cumplir que:

- La función $f(x)$ tenga un número finito de máximos y mínimos. Obsérvese que es más fácil saber sobre qué ancho de banda se concentra la energía de una señal analizándola en el dominio de la frecuencia.

- Que sea absolutamente integrable en el intervalo $(-\infty, \infty)$.

Por definición se tiene que la Transformada de Fourier es la operación matemática que asocia a una función $f(x)$, real o compleja, de variable real $x$, su espectro $\mathfrak{J}\{f(x)\}=F(u)$; esta se puede definir por medio de la integral:

$$
\Im\{f(x)\}=F(u)=\int_{-\infty}^{\infty} f(x) \cdot e^{-2 \pi x u i} d x
$$

Cabe anotar que la transformada de Fourier genera implícitamente un principio de incertidumbre de Heisemberg [5].

\section{A. Reciprocidad de la transformada de Fourier}

Asumiendo que la Transformada de Fourier de la función $f(x, y)$ se puede escribir la transformada inversa de Fourier como:

$$
f(x, y)=\mathfrak{I}^{-1}\{F(u, v)\}
$$

De lo anterior se concluye que la transformada de Fourier se define como el operador $\mathfrak{J}$, que a su vez es un operador lineal.

$$
f(x, y)=\mathfrak{\Im}\left\{\mathfrak{J}^{-1}[f(x, y)]\right\}=\mathfrak{\Im}^{-1}\{\mathfrak{\Im}[f(x, y)]\}
$$

Esta propiedad se puede demostrar desde la manera integral [6]. 


\section{Generación de ruido a partir de la estructura química de la materia}

Para determinar la estructura de las moléculas, el método predilecto en la química orgánica es la espectroscopia de Resonancia Magnética Nuclear (RMN), ya que complementa los resultados de otras espectroscopías como la de masas (que indica la fórmula y el tamaño molecular) y la de infrarrojo (que identifica los grupos funcionales) [8]; las aplicaciones de la espectroscopía RMN va desde la determinación de estructuras de moléculas pequeñas hasta el desarrollo de técnicas avanzadas para el estudio del plegamiento de las proteínas y de los mecanismos de reacción en procesos complejos como los bioquímicos, por ejemplo los fotosintéticos [9], o en la determinación cuantitativa de las especies absorbentes [10].

La orientación de los espines magnéticos de los núcleos de los átomos que conformen cierta estructura, deja de ser aleatoria cuando dichos núcleos son sometidos a la presencia de un campo magnético, ya que toman cierto posicionamiento, alineándose en contra (antiparalelo) o a favor (paralelo) respecto al campo externo, donde las orientaciones paralelas corresponden a estados de menor energía, y cuyo valor depende de la intensidad del campo magnético. Luego, dichos núcleos se someten a RF, radiación que absorben, tal que pasan de un estado de menor energía a uno de mayor energía invirtiendo su espín [8]; cuando ocurre esta situación se dice que los núcleos están en «resonancia» con la radiación aplicada, y la frecuencia exacta de dichas ondas electromagnéticas depende no solo de la intensidad del campo, sino también de la identidad de los núcleos, de los cuales los que han sido de mayor interés para la elucidación de estructuras químicas y bioquímicas son ${ }_{1}^{1} \mathrm{H},{ }_{6}^{13} \mathrm{C},{ }_{7}^{15} \mathrm{~N},{ }_{9}^{19} \mathrm{~F}$ y ${ }_{15}^{31} \mathrm{P}$ y , especies que al tener número masa impar presentan un número cuántico de espín de 1/2 [10,11] , dando así lugar a fenómenos magnéticos.

En el momento de aplicar un campo magnético externo, los electrones que se mueven en torno a los núcleos en las moléculas también generan «pequeños» campos magnéticos locales opuestos al externo, de tal forma que el campo efectivo es menor al aplicado, lo que se conoce como efecto de apantallamiento, lo que genera el llamado desplazamiento químico, que está relacionado con la densidad electrónica [12]; de acuerdo a la configuración molecular, los núcleos están protegidos por configuraciones o nubes electrónicas diferentes, lo que permite detectar distintas señales características en la RMN para cada caso, como se puede observar en los espectros de RMN. Entonces la frecuencia de radiación de radiofrecuencias absorbida por un núcleo determinado es afectada intensamente por su entorno molecular, es decir, los núcleos y electrones vecinos; cuando los momentos magnéticos de los núcleos adyacentes interaccionan entre sí, se produce un acoplamiento magnético que modifica la distribución electrónica de los enlaces químicos, lo que se denomina interacción de polarización [10], la que constituye en sí una interacción hiperfina entre espines tanto del núcleo como del electrón, lo que permite determinar la ubicación de núcleos dentro de la configuración [9]. Los desplazamientos químicos de las señales de $\mathrm{RMN}$ indican información acerca de la naturaleza tanto de los hidrógenos como de los carbonos: la integración de señales y el acoplamiento espín-espín que se observa en los espectros señalan el número de hidrógenos y de carbonos, y su distribución dentro de la estructura. De esta manera, las técnicas de pulsos y Transformadas de Fourier generan 
información configuracional más directa y presentan grandes ventajas para elucidar estructuras nuevas relacionadas a algunas de referencia conocidas [13]. De hecho, la espectroscopía RMN puede analizar estructuras muy complejas como es el caso de las proteínas, por lo que compite de esta manera con la cristalografía de rayos X [14].

Como se puede observar, la RMN consta básicamente de los procesos de orientación, absorción, excitación y finalmente la relajación de los núcleos, etapa en la cual estos recaen a su estado original -de menor energía-, emitiendo así la energía encapsulada en la señal en el dominio del tiempo, que se denomina señal caída libre de inducción. La relajación ocurre por dos mecanismos diferentes e independientes: el relajamiento longitudinal debido a las interacciones espín-red (núcleo-molécula) lleva a un aumento de la amplitud de las vibraciones o rotaciones térmicas, es decir, de temperatura; mientras que el relajamiento transversal es debido a las interacciones espín-espín (núcleo-núcleo), ocurriendo un intercambio de energía entre los núcleos de tal manera que unos alcanzan frecuencia de precesión mayor, y otros menor, a la frecuencia de Larmor. [10,12]. Estos mecanismos repercuten para que el proceso de RMN sea relativamente lento frente a otras técnicas como por ejemplo la espectroscopía infrarroja, ya que el cambio en la amplitud vibracional al absorber las RF es de aproximadamente $1 \mathrm{~ms}$, en tanto, con la radiación infrarroja se considera instantáneo, de alrededor de $0,1 \mathrm{ps}$; entonces cuando se presenten dos especies que se interconviertan más rápido que el tiempo de RMS, solo se registrará un espectro promedio, en vez de espectros distintos para cada configuración, lo que genera un efecto de borrosidad, el cual puede servir para estudiar la velocidad y las energías de activación de procesos muy rápidos [8]; sin embargo, el evidente ruido superpuesto al modelo de caída libre de inducción es un artefacto experimental causado por la bandas laterales giratorias, debe considerarse cuando la señal es convertida desde el dominio del tiempo al de la frecuencia a través de la Transformada de Fourier. El resultado de estas interferencias sobre la señal emitida es el ruido que afecta la calidad de la imagen.

\section{Resultados}

Usando un criterio de medida cuantitativo, se calcula la relación Señal-Ruido, $R S R$ a través de la siguiente expresión:

$$
R S R=\frac{S}{\sigma}
$$

Donde:

$S:$ Valor medio de la intensidad de señal de una región de interés centrada en la imagen.

$\sigma$ : Desviación estándar del fondo de la imagen.

Este resultado es la ganancia, es de 1/7, lo que significa un aumento de alrededor del $14 \%$ de la relación, que de otra forma se puede considerar como un $14 \%$ de la reducción de ruido. 
Para analizar como varía la relación señal-ruido, se realizó el análisis de los patrones de intensidad de la señal, como se puede ver en la Figura 3, donde se muestra la imagen original de RMN con ruido:
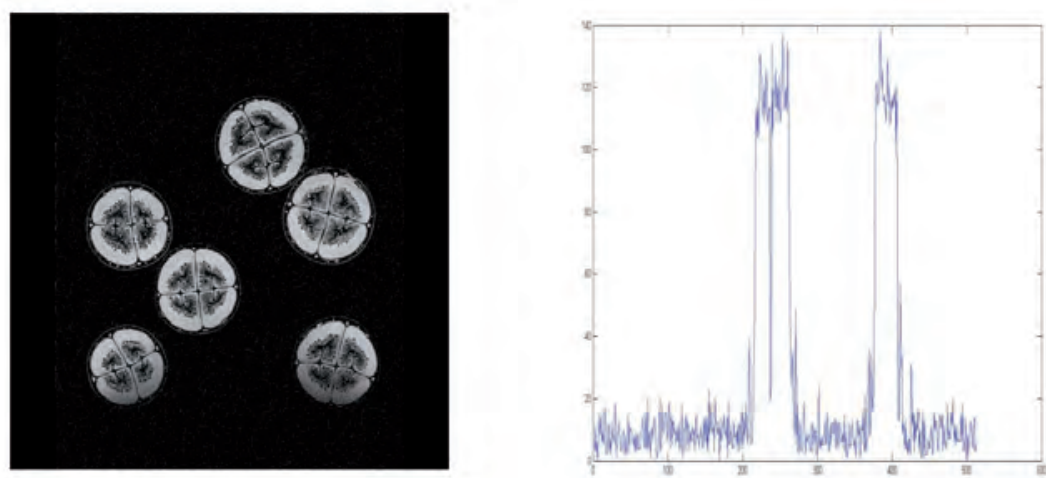

Figura 3. Resonancia magnética y espectro de intensidades de lulos sin filtro.

Ahora, para filtrar la señal se utilizó una función rectángulo, lo que permite mejorar la resolución de la imagen de los lulos como se puede visualizar en la Figura 4:
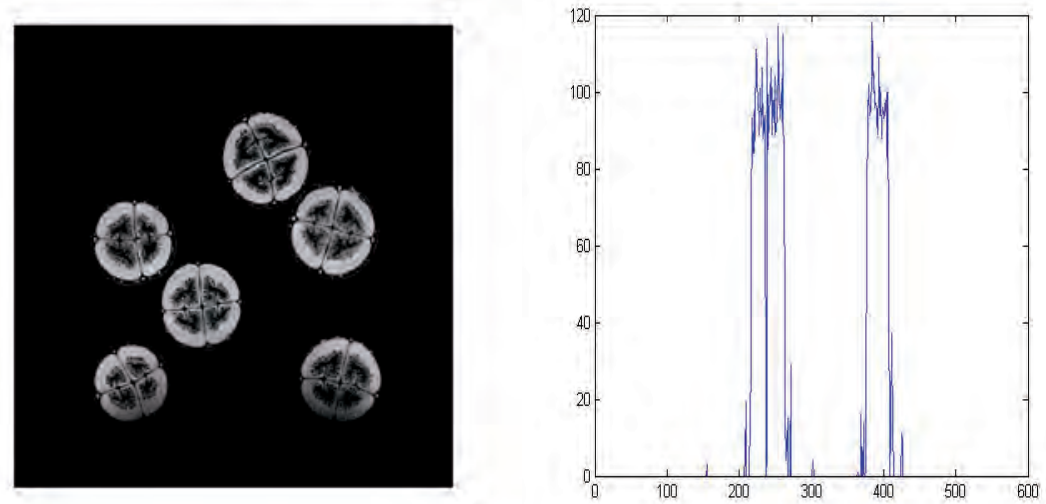

Figura 4. Resonancia magnética y espectro de intensidades de lulos con filtro.

Aplicando el filtro a otras imágenes médicas como la mostrada en la Figura 5, se observa una reducción del nivel de ruido de la señal, lo que se traduce en mejoramiento de la calidad de la imagen obtenida, ya que se corrigen defectos como la granularidad.
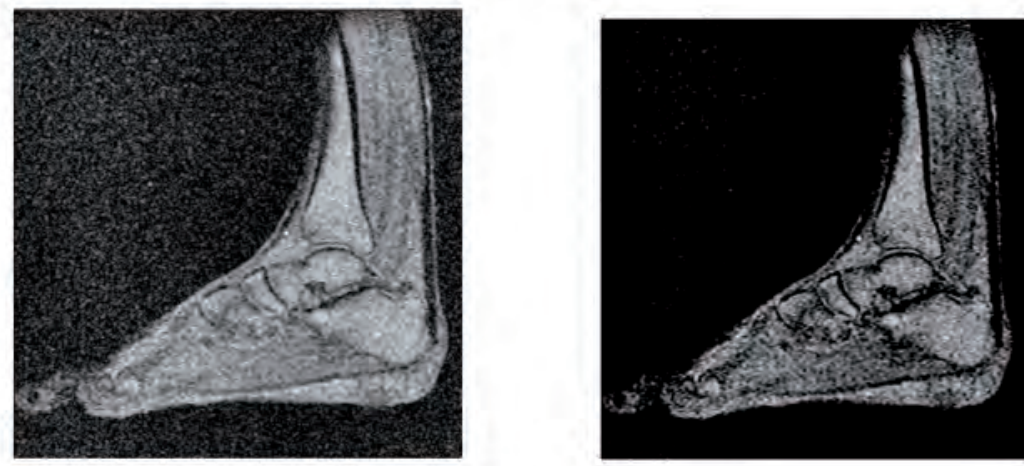

Figura 5. Resonancia magnética de un pie sin filtro (izquierda) y con filtro (derecha). 


\section{Conclusiones}

Últimamente la RMN se ha venido aplicando en otras áreas distintas a la química, como por ejemplo la ingeniería, el control de calidad, la medicina y la biología, principalmente a través de la obtención de imágenes. Entre las ventajas de obtener dichas imágenes de los objetos por RMN se tienen: principalmente es una técnica de naturaleza no invasiva, es decir, no hay potencial efecto de daños a los objetos, sobre todo los de origen biológico; es además una técnica no reproducible; también es importante la velocidad de adquisición de datos; y finalmente su alta resolución espacial. Inicialmente, la principal desventaja de dicha tecnología es el costo, pero su creciente utilización por sus inherentes ventajas ha llevado a desvirtuar esta aparente barrera.

Para el diagnóstico médico las imágenes por RMN son un pilar fundamente por su cualidad no invasiva, debido a que los núcleos de hidrógeno o protones son muy abundantes en el organismo humano, por lo que se pueden detectar, por ejemplo, lesiones en tejidos blandos con un alto grado de fidelidad que no se puede obtener con otros medios; pero también las imágenes de RMI se han implementado en tareas diversas de la industria, el comercio y la ciencia, como en el sector alimentario, para el examen no invasivo de productos: determinación del contenido de lípidos y humedad tanto en las emulsiones carne/agua como en aceites comestibles; análisis de la transmisión de calor sobre la estructura y composición de los alimentos durante los tratamientos térmicos; estudio de la cristalización de las grasas; la descripción cuantitativa de los fenómenos de transporte durante el procesado; en los cárnicos, permite explorar la distribución de grasa y músculos; la visualización de la hidratación de los alimentos; la evaluación del curado de la carne de cerdo; entre muchos más casos. En otros sectores permite el examen de la filtración de partículas a partir de núcleos de arenas; o en polímeros, la investigación de su estructura.

Las imágenes por RMN se han convertido en la herramienta imprescindible en la investigación no invasiva de materiales, debido principalmente a: la búsqueda de protocolos prácticos y sofisticados de refinamiento de datos; la construcción de secuencias de señales de impulsos; y los procedimientos de adquisición de datos, en particular, para la identificación de núcleos diferentes al hidrógeno.

Para mejorar la resolución de las imágenes por medio de la reducción de ruido (que se genera de acuerdo a lo descrito en la sección 4 del presente trabajo) se aplican sobre las señales filtros usando transformaciones matemáticas que generan los algoritmos que a través de la potencia de ruido estimada logran discriminar el nivel de ruido respecto al nivel de la señal, lo que se conoce como RSR. En el caso de estudio se usa un filtro basado Transformada de Fourier con un rectángulo de borde que permitió un incremento del 14\% en la Relación Señal Ruido, RSR.

Sin embargo, usando estos filtros pueden presentarse un suavizamiento de picos en el espectro debido a una pérdida de detalles finos en la señal, por lo que dicha situación puede ser corregida usando otro tipo de transformadas matemáticas diferentes a las de 
la Teoría de Fourier, por ejemplo, la Transformada Wavelet [15], las cuales se encuentran actualmente en desarrollo.

\section{Referencias}

[1] F. Bloch, "Nuclear induction". Phys. Rev. n. ${ }^{\circ}$ 70, vol. 7-8, pp. 460-474. 1946.

[2] P.C. Lauterbur Image Formation by Induced Local Interactions: Examples Employing. Nuclear Magnetic Resonance. Nature, n. ${ }^{\circ} 242$, pp. 190-191. 1973.

[3] J. T. Vaugham y collaborators, $7 T$ Vs 4T: Power, Homogeneity and Signal to Noise Comparison in Head Images MRM; n. 46, 24-30. 2001.

[4] J. T. Abduljilil, A. Kangarlu, X. Zhang, Acquisiton of Human Multislise MR Images at 8T JCAT, 23(3)-335-340 May-Jun 1999.

[5] H. Dym, H. p. McKean, Fourier Series and Integrals. Academic Press, New York, 1972.

[6] W. Rudin, Analisis Real y Complejo. Madrid: Alhambra, 1979.

[7] J. Gili, Introducción Biofísica a la resonancia magnética aplicada a la clínica. Barcelona, junio de 2009.

[8] J. McMurry, Química Orgánica Cengage Learning Editores, 2/01/2008 - 1352 pp. 440-481.

[9] H. Martina. "Introduction to Magnetic Resonance Methods in Photosynthesis». Photosynthesis Research n. ${ }^{\circ} 102$, pp. 305-310. 2009

[10] D. Skoog, F. J. Haller, y T. A. Nieman, Principio de Análisis Instrumental. 5a . Ed., España: Mc. Graw-hill, 2001 , pp. 481 -536.

[11] W. F. Pickering, Química Analítica Moderna. 1976, 688 páginas.

[12] M. H. Levitt, Spin Dynamics: Basics of Nuclear Magnetics Resonance. Wiley-Blackwell (an imprint of John Wiley \& Sons Ltd); Edición: 2nd Revised edition (7 de marzo de 2008), 744 páginas. Capítulo 3: NMR Spectroscopy. pp. $39-59$.

[13] D. Marcano, y M. Hasegawa, Fitoquímica Orgánica. Universidad Central de Venezuela, Consejo de Desarrollo Científico y Humanística. 2002. pp. 81-82.

[14] J. M. Miranda, J. L. Sebastián, M. Sierra, y Margineda, José. Ingeniería de Microondas: Técnicas Experimentales Pearson Educación, 2002 - 365 páginas, Pág. 20.

[15] J.E. Paz, Disminución del Nivel de Ruido en Imagénes de Resonancia Magnética Usando la Transformada Wavelet. Memorias II Congreso Latinoamericano de Ingeniería Biomédica, La Habana (C uba) 2001. Centro de Estudios de Electrónica y Tecnologías de la Información, CEETI. 